\title{
OPTIMAL DESIGN OF CONIC-CYLINDRICAL GEAR REDUCTION UNIT USING FUZZY PHYSICAL PROGRAMMING
}

\author{
Hong-Zhong Huang ${ }^{1,2}$, Xu Zhang ${ }^{1}$ Zhi-Gang Tian ${ }^{1}$ \\ Chun-Sheng $\mathrm{Liu}^{2}$ and Ying-Kui Gu${ }^{1}$ \\ 'School of Mechanical Engineering, Dalian University of Technology, Dalian, 116023, China
}

${ }^{2}$ Department of Mechanical Engineering, Heilongjiang Institute of Science and Technology, Harbin, 150027, China

Abstract: Conic-cylindrical gear reduction unit as a high-performance power transmission device is widely used to build various machineries. There are lots of fuzzy factors in its manufacturing process and operation environment, which should be taken into consideration in the design process. Fuzzy physical programming is an effective multiobjective optimization method which incorporates fuzziness in its problem formulation. The fuzzy physical programming model for the optimal design of two-stage conic-cylindrical gear reduction unit is developed in this paper, and genetic algorithm is used to solve the model. An example is given to illustrate that fuzzy physical programming can consider the fuzziness of conic-cylindrical gear reduction unit substantially, and conforms more perfectly to the engineering realities.

Key words: conic-cylindrical gear reduction unit, multiobjective optimization, physical programming, fuzzy physical programming

\section{INTRODUCTION}

Generally, mechanical design has been taken as the multiobjective problem and the design process is actually an optimizing process, considering multi-restricted conditions. Multiobjective optimization has been applied widely in the field of mechanical design. In recent years, some new algorithms for the multiobjective optimization appear, such as collaborative 
optimization $^{[1]}$, interactive multi-objective optimization ${ }^{[2]}$, physical programming $^{[3]}$ and VEGA (Vector Evaluated Genetic Algorithm) ${ }^{[4]}$. These algorithms have characteristics of their own, and have found applications in various engineering practical problems.

There are lots of fuzzy factors in manufacture process and operation environment ${ }^{[5]}$. The key sources of fuzziness are as follows: the complexity of system's structure and mechanism; the limitation of test conditions; the limitation and subjectivity of human being. Because of the existence of fuzziness, the product performances (weight, price, volume, etc.) are thus fuzzy sets.

Fuzzy physical programming is a new efficient multiobjective optimization method $^{[6]}$, which inherits the advantages of physical programming and considers the fuzziness of multiobjective systems. Fuzzy physical programming can solve fuzzy multiobjective design problems and get the design results considering fuzzy factors by incorporating fuzziness in design variables, objective functions and constraints.

Conic-cylindrical gear reduction unit as a high-performance power transmission system is widely used to make various machineries, and its structure and performance has distinct influence on robust, noise level, bearing capacity and service life of the whole machinery. Currently, there are rare researches in this area. Regarding geometry structure and bearing capacity are as main optimization objectives, and considering the fuzziness of the design variables and objectives substantially, this paper develops the fuzzy physical programming model for optimal design of conic-cylindrical gear reduction unit. Genetic Algorithms is applied to solve the formulated fuzzy physical programming model.

\section{FUZZY PHSICAL PROGRAMMING}

\subsection{PHSICAL PROGRAMMING}

Physical programming is a new effective multicriteria optimization method first brought forward by Achille Messac in $1995^{[7]}$, which reduces the computational intensity of large problems and places the design process into a more flexible and natural framework. It can capture the designer's physical understanding of the desired design outcomes by forming the aggregate objective function. Designers specify ranges of different degrees of desirability (desirable, tolerance, undesirable, etc.) for each design metric. 
Once the designers' preferences are articulated, obtaining the corresponding optimal design is a non-iterative process.

Design objectives are classified into four different categories. Each class comprises two cases, hard and soft, subject to the sharpness of preference. The qualitative meaning of soft preference function is depicted in Figure $1^{[8]}$. The value of the objective function, $g_{i}$, is on the horizontal axis, and the corresponding preference function, $\bar{g}_{i}$, is on the vertical axis. No matter which category, the smaller the value of preference functions is, the better it is.

\subsection{Mathematical model of fuzzy physical programming ${ }^{[6]}$}

With fuzzy parameters $X$, the design objective value of fuzzy system, $\widetilde{g}_{i}(X)$, is a fuzzy set. Supposing that $\widetilde{g}_{i}(X)$ is represented using normal membership function, and takes the form

$$
\mu_{\tilde{g}_{i}(x)}\left(g_{i}\right)=e^{-\left[\frac{g_{i}-g_{i}(x)}{\delta_{i}}\right]^{2}}, \delta_{i}>0
$$

$f p_{i}\left(\widetilde{g}_{i}(X)\right)$ is used to represent the preference function with respect to fuzzy set $\widetilde{g}_{i}(X)$. Referring to the definition of satisfying degree function for generalized fuzzy constraint ${ }^{[5]}, f p_{i}\left(\widetilde{g}_{i}(X)\right)$ takes the form

$$
f p_{i}\left(\widetilde{g}_{i}(X)\right)=\frac{\int_{g_{i}(X)-3 \delta_{i}}^{g_{i}(X)+3 \delta_{i}} \bar{g}_{i}\left(g_{i}\right) \cdot \mu_{\tilde{g}_{i}(X)}\left(g_{i}\right) \mathrm{d} g_{i}}{\int_{g_{i}(X)-3 \delta_{i}}^{g_{i}(X)+3 \delta_{i}} \mu_{\tilde{g}_{i}(X)}\left(g_{i}\right) \mathrm{d} g_{i}}
$$

The fuzzy aggregate objective function, $f p_{i}\left(\widetilde{g}_{i}(X)\right)$, is formulated by synthesizing the fuzzy preference functions for all the design metrics

$$
f p(X)=\log _{10}\left\{\frac{1}{n_{s c}} \sum_{i=1}^{n_{s c}} f p_{i}\left(\widetilde{g}_{i}(X)\right)\right\}
$$



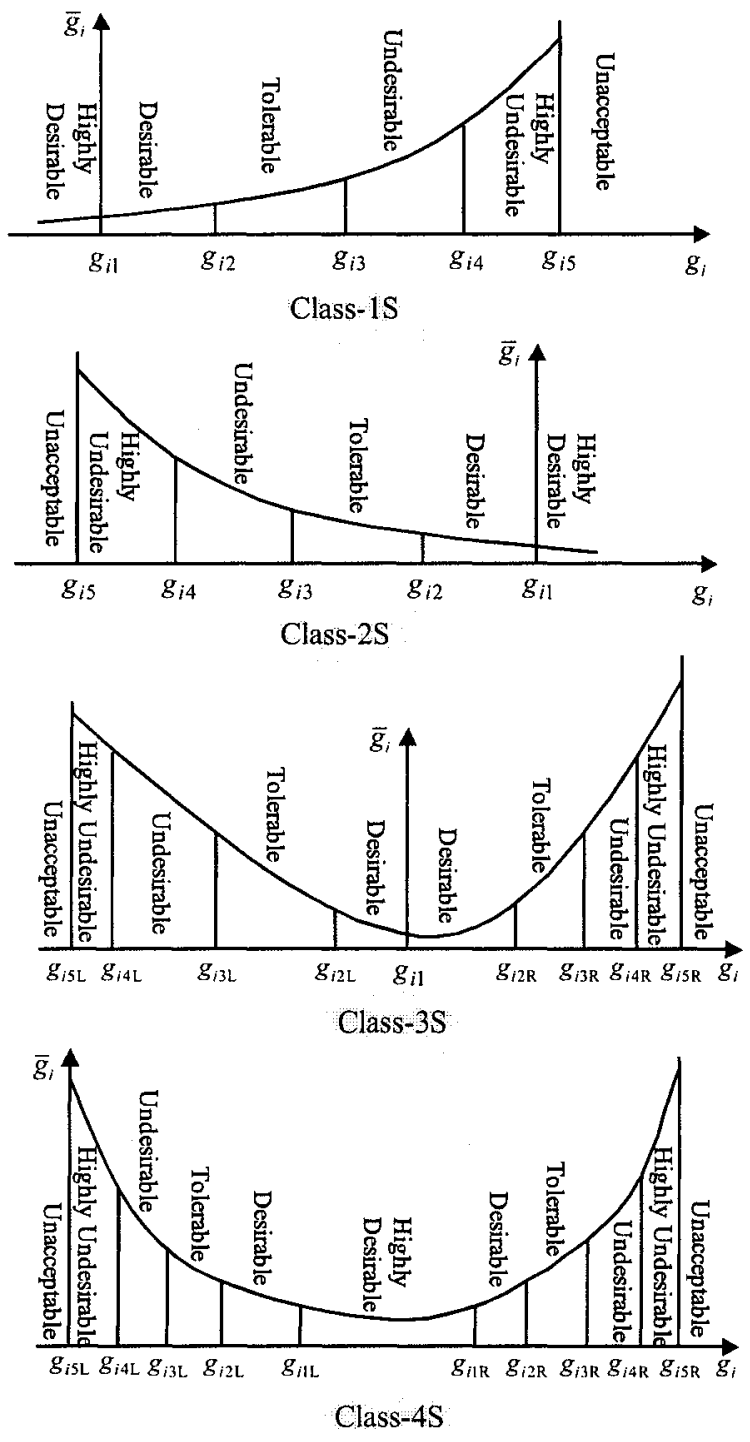

Figure 1. Preference function ranges for $i$ th generic metric

With the fuzzy aggregate objective function described above, the fuzzy physical programming problem model takes the following form ${ }^{[6]}$ 


$$
\begin{aligned}
& \min _{X} f p(X)=\log _{10}\left\{\frac{1}{n_{s c}} \sum_{i=1}^{n_{s c}} f p_{i}\left(\widetilde{g}_{i}(X)\right)\right\} \quad \text { (for soft classes) } \\
& \text { st. } \quad g_{i}(X) \leq g_{i 5} \quad \text { (for class } 1-\mathrm{S} \text { ) } \\
& g_{i}(X) \geq g_{i 5} \quad \text { (for class2 - S) } \\
& g_{i 5 L} \leq g_{i}(X) \leq g_{i 5 R} \quad \text { (for class } 3-\mathrm{S} \text { and } 4-\mathrm{S} \text { ) } \\
& g_{i}(X) \leq g_{i M} \quad \text { (for class } 1-\mathrm{H} \text { ) } \\
& g_{i}(X) \geq g_{\text {im }} \quad \text { (for class2 - H) } \\
& g_{i m} \leq g_{i}(X) \leq g_{i M} \quad \text { (for class } 3-\mathrm{H} \text { and } 4-\mathrm{H} \text { ) } \\
& x_{j m} \leq x_{j} \leq x_{j M}
\end{aligned}
$$

where $g_{i m}, g_{i M}, x_{j m}$ and $x_{j M}$ represent minimum and maximum values, and $n_{s c}$ is the number of soft design metrics that the problem comprises. The process of formula transforming is similar to that in physical programming problem model ${ }^{[8,9]}$.

\subsection{Computational procedure of fuzzy physical programming}

The solution to the multiobjective optimization problem using the proposed fuzzy physical programming approach can be determined using the following step-by step procedure.

a) Determine design objectives and design variables.

b) Specify the membership function for each design metric.

c) Specify the class type for each design metric (class 1S-4H).

d) Provide the range limits for each design metric.

e) Form the fuzzy physical programming problem model.

f) Solve the problem model, and obtain the optimal design.

In conventional optimization methods, multiobjective is converted into single objective, which only can obtain local optimum. In this paper, Genetic Algorithms is used in solving the formulated fuzzy physical programming model, and has demonstrated its ability to obtain the global optimum even if there exists local optimums.

\section{EXAMPLE}

In the section, the case of two-stage conic-cylindrical gear reduction unit, which is used in chain moving machinery, is studied using fuzzy physical programming approach. A pair of conic gears is used as high-speed gears, while a pair of cylindrical gears is used as the low-speed gears. The 
performance of fuzzy physical programming is compared with that of physical programming. The objective is to minimize volume and minimize difference of power delivered between high-speed gear and low-speed gear $[10,11]$

\subsection{Problem formulation}

The design vector $\boldsymbol{x}$ is

$$
\boldsymbol{x}=\left[m_{1}, \psi_{R}, Z_{1}, m_{2}, \psi_{a}, Z_{3}, i_{1}, \beta\right]
$$

where $m_{i}$ is the module value of the $i$ th gear set, $Z_{i}$ represents the tooth numbers of the $i$ th pinion, $\psi_{R}$ is the face width of the conic gear set, $\psi_{a}$ is the face width of the cylindrical gear set, $i_{1}$ represents the gear ratio of the high-speed gear, $\beta$ is the helix angle. The values of the primary design parameters refer to reference 10 .

The objective of the minimum volume of the gear reduction unit takes

$$
V=0.392669\left[m_{1}^{3} \psi_{R} Z_{1}^{2}\left(1-\psi_{R}+\frac{\psi_{R}^{2}}{3}\right)\left(i+i_{1}^{2}\right)+m_{2}^{3} \psi_{a} Z_{3}^{3}\left(1+\frac{i}{i_{1}}\right)\left(1+\frac{i^{2}}{i_{1}^{2}}\right) \frac{1}{\cos ^{3} \beta}\right]
$$

where $i$ is the general gear ratio.

The objective of minimum difference of power delivered between highspeed gear and low-speed gear is represented by

$$
\Delta P=\left|P_{h}-P_{l}\right|
$$

where

$$
\begin{gathered}
P_{h}=\frac{\psi_{R}\left(1+0.5 \psi_{R}\right)^{2} i_{1} m_{1}^{3} Z_{1}^{3}}{Z_{E}^{2} Z_{H}^{2} k} \cdot \frac{n_{1}[\sigma]_{H L}^{2}}{4 \times 9.55 \times 10^{6}} \\
P_{l}=\frac{\psi_{a} m_{2}^{3} Z_{3}^{3} i}{k Z_{E}^{2} Z_{H}^{2} Z_{E}^{2} i_{1}^{2} \cos ^{3} \beta} \cdot \frac{n_{1}[\sigma]_{H L}^{2}}{4 \times 9.55 \times 10^{6}}
\end{gathered}
$$

The mathematical model of the multi-stage gear reduction unit optimization problem is formulated as follows:

minimize $V, \operatorname{minimize} \Delta P$ 
$0 \leq i_{1} \leq 5$

$8 \pi / 180 \leq \beta \leq 25 \pi / 180$

$0.2 \leq \psi_{R} \leq 0.33$

$0.4 \leq \psi_{a} \leq 0.6$

$Z_{1}-17 m_{1} / \sqrt{1+i_{1}^{2}} \geq 0$

$Z_{2}-17 \cos ^{3} \beta \geq 0$

$\frac{1}{2 \pi}\left\{\frac{Z_{1}}{\cos \left(\tan ^{-1}\left(1 / i_{1}\right)\right)}\left[\tan \left(\cos ^{-1} \frac{Z_{1} \cos (\pi / 9)}{Z_{1}+2 \cos \left(\tan ^{-1}\left(1 / i_{7}\right)\right)}\right)\right]+\right.$
$\left.\frac{Z_{1} i_{1}}{\sin \left(\tan ^{-1}\left(1 / i_{1}\right)\right)}\left[\tan \left(\cos ^{-1} \frac{Z_{1} i_{1} \cos (\pi / 9)}{Z_{1} i_{1}+2 \sin \left(\tan ^{-1}\left(1 / i_{1}\right)\right)}\right)-\tan (\pi / 9)\right]\right\}-1.6 \geq 0$

$\left[1.88-3.2\left(\frac{1}{Z_{3}}+\frac{i_{1}}{i Z_{3}}\right)\right] \cos ^{3} \beta-1.3 \geq 0$

$\frac{\psi_{a} Z_{3}\left(1+i / i_{1}\right) \sin \beta}{2 \pi m_{2}}-1 \geq 0$ 


$$
\begin{aligned}
& {[\sigma]_{H L}^{2}-\frac{4 k T_{1} Z_{E}^{2} Z_{H}^{2}}{\psi_{R}\left(1-0.5 \psi_{R}\right)^{2} m_{1}^{3} Z_{1}^{3} i_{1}} \geq 0} \\
& 1-\frac{4 k T_{1} Y_{F_{1}} Y_{S_{1}}}{\psi_{R}\left(1-0.5 \psi_{R}\right)^{2} m_{1}^{3} Z_{1}^{2} \sqrt{1+i_{1}^{2}}[\sigma]_{F_{1}}} \geq 0 \\
& 1-\frac{4 k T_{1} Y_{2_{1}} Y_{S_{2}}}{\psi_{R}\left(1-0.5 \psi_{R}\right)^{2} m_{1}^{3} Z_{1}^{2} \sqrt{1+i_{1}^{2}}[\sigma]_{F_{2}}} \geq 0 \\
& 1-\frac{Z_{E} Z_{H} Z_{\varepsilon}}{[\sigma]_{H L}} \sqrt{\frac{2 k T_{2}\left(2+\psi_{R}\right) \cos ^{3} i_{1}}{m_{2}^{3} Z_{2}^{3} \psi_{R} \sqrt{1+i_{1}^{2}}}\left(1+\frac{i_{1}}{i}\right)} \geq 0 \\
& 1-\frac{2 k T_{2}\left(2-\psi_{R}\right) \cos ^{3} \beta}{m_{2}^{3} Z_{2}^{2} \psi_{R} \sqrt{1+i_{1}^{2}}[\sigma]_{F_{3}}} Y_{F_{3}} Y_{S_{3}} Y_{\beta_{3}} Y_{t_{3}} \geq 0 \\
& 1-\frac{2 k T_{2}\left(2-\psi_{R}\right) \cos ^{3} \beta}{m_{2}^{3} Z_{2}^{2} \psi_{R} \sqrt{1+i_{1}^{2}}[\sigma]_{F_{4}}} Y_{F_{4}} Y_{S_{4}} Y_{\beta_{4}} Y_{t_{4}} \geq 0
\end{aligned}
$$

Eq. (7) is the range of the gear ratio, Eq. (8) is the range of the helix angle, Eqs (9)-(10) reflect the face width constraints, Eqs (11)-(12) represent the interference constraints, Eqs (13)-(15) represent the limit of overlap ratio, and Eqs (16)-(21) represent the strength constraints.

\subsection{Results and discussions}

Subject to the foregone conditions, we can establish the fuzzy physical programming problem model as Eq. (4). Region limits and the parameter $\delta_{i}$ of the design objections are show in Table 1. Genetic algorithms is used to solve the formulated model, and the optimal results are obtained and depicted in Table 2 and 3 . The deviation between the result of fuzzy physical 
programming and physical programming is shown in Table 4. Considering the fuzzy factors of the system, the largest deviations of design objective and design variable are $5.9 \%$ and $13.2 \%$, respectively. It is obvious that neglecting the fuzzy factors in the system will not lead to true optimal solution.

Table 1 Region limits and the parameter $\delta_{i}$ of the design objections

\begin{tabular}{lllllllll}
\hline $\begin{array}{l}\text { Design } \\
\text { objective }\end{array}$ & $\begin{array}{l}\text { Type of preference } \\
\text { function }\end{array}$ & $g_{i 5}$ & $g_{i 4}$ & $g_{i 3}$ & $g_{i 2}$ & $g_{i 1}$ & $\delta_{i}$ \\
\hline$V\left(\mathrm{~m}^{3}\right)$ & $1-\mathrm{S}$ & 0.020 & 0.018 & 0.016 & 0.014 & 0.010 & $-\infty$ & 0.0000280 \\
$\Delta P(\mathrm{~kW})$ & $1-\mathrm{S}$ & 0.025 & 0.024 & 0.022 & 0.019 & 0.015 & $-\infty$ & 0.0000420 \\
\hline
\end{tabular}

Table 2 The design variables' value

\begin{tabular}{lllllllll}
\hline Design variable & $m_{1}$ & $\psi_{R}$ & $Z_{1}$ & $m_{2}$ & $\psi_{a}$ & $Z_{2}$ & $i_{1}$ & $\beta$ \\
\hline FPP & 3.849 & 0.203 & 19.817 & 3.675 & 0.400 & 21.379 & 4.76 & 0.140602 \\
PP & 3.856 & 0.208 & 19.923 & 3.774 & 0.425 & 21.220 & 4.72 & 0.140855 \\
\hline
\end{tabular}

Table 3. The design objective functions' value

\begin{tabular}{lll}
\hline Design objective & $V\left(\mathrm{~m}^{3}\right)$ & $\Delta P(\mathrm{~kW})$ \\
\hline FPP & 0.0125 & 0.0175 \\
PP & 0.0144 & 0.0182 \\
\hline
\end{tabular}

Table 4. The deviation between the result of fuzzy physical programming and physical programming $(\%)$

\begin{tabular}{lcccccccccc}
\hline $\begin{array}{l}\text { Design variable and } \\
\text { Design objective }\end{array}$ & $m_{1}$ & $\psi_{R}$ & $Z_{1}$ & $m_{2}$ & $\psi_{a}$ & $Z_{2}$ & $i_{1}$ & $\beta$ & $V$ & $\Delta P$ \\
\hline Deviation & 1.2 & 2.4 & 0.5 & 2.6 & 5.9 & 0.7 & 5.7 & 0.2 & 13.2 & 3.8 \\
\hline
\end{tabular}

\section{CONCLUSIONS}

The fuzzy physical programming model for the optimal design of twostage conic-cylindrical gear reduction unit is developed in this paper, and genetic algorithm is used to solve the model. Compared with the physical programming approach, fuzzy physical programming is a more reasonable method for complex engineering systems. Combining with genetic algorithms, fuzzy physical programming approach can obtain the global optimum. The example illustrates that fuzzy physical programming can consider the fuzziness of conic-cylindrical gear reduction unit substantially, and conforms more perfectly to the engineering realities. 


\section{REFERENCES}

1. Tappeta R V, Renaud J E. Multiobjective collaborative optimization. ASME, Journal of Mechanical Design, 1997, 119(9): 403 411

2. Tappeta $\mathbf{R}$ V, Renaud J E. Interactive multi-objective optimization design strategy for decision based design. ASME, Journal of Mechanical Design, 2001, 123(6): 205 215

3. Messac A, Gupta S, Akbulut B. Linear physical programming: A new approach to multiple objective optimization. Transactions on Operational Research, 1996, 8: 39 59

4. Tapabrata R, Kang T, Kian C S. Multiobjective design optimization by an evolutionary algorithm. Engineering Optimization, 2001, 33(4): 399 424

5. Huang H-Z. Fuzzy design. Beijing: China Machine Press, 1999

6. Tian Z-G, Huang H-Z, Guan L-W. Fuzzy physical programming and its application in optimization of through passenger train plan. Proceedings of the Conference on Traffic and Transportation Studies, ICTTS, 2002, 1: 498 503

7. Messac A, Hattis P D. High speed civil transport (HSCT) plane design using physical programming. AIAA/ASME/ASCE/AHS Structures, Structural Dynamics \& Material Conference - Collection of Technical Papers. 1995, 3: 10 13

8. Messac A. Physical programming: Effective optimization for computational design. AIAA Journal, 1996, 34(1): 149 158

9. Huang $\mathrm{H}-\mathrm{Z}$, Tian $\mathrm{Z}-\mathrm{G}$, Guan L W. Neural networks based interactive physical programming and its applications in mechanical design. Chinese Journal of Mechanical Engineering, 2002, 38(4): 51 57

10. Wei $\mathrm{H}$, Zhang J-S. The multi-aim optimization design for conic-cylindrical reducer. Journal of Lianyungang College of Chemical Technology, 1999, 12(1): 11 14

11. Huang H-Z. Mechanical fuzzy optimization: Principle and application. Beijing: Science Press, 1997 